\title{
Shenzhi Jiannao Formula Ameliorates Vascular Dementia in Vivo and in Vitro by Inhibition Glutamate Neurotoxicity via Promoting Clathrin- Mediated Endocytosis
}

\section{Danfeng Tian}

Beijing University of Chinese Medicine https://orcid.org/0000-0002-8371-3887

\section{Yangyang Guo}

Beijing University of Chinese Medicine

\section{Dandan Zhang}

Beijing University of Chinese Medicine

\section{Qiang Gao}

Beijing University of Chinese Medicine

Ganlu Liu

Beijing University of Chinese Medicine

Jingfeng Lin

Beijing University of Chinese Medicine

\section{Ze Chang}

Beijing University of Chinese Medicine

\section{Yuchun Wang}

Beijing University of Chinese Medicine

\section{Rui Su}

Beijing Hospital of Traditional Chinese Medicine

Zhenyun Han ( $\nabla$ tohanzhenyun@126.com )

Shenzhen Hospital of Beijing University of Chinese Medicine (Longgang) https://orcid.org/0000-00026831-2364

\section{Research}

Keywords: Shenzhi Jiannao Formula, Vascular dementia, Clathrin-mediated endocytosis, Glutamate, Neurotoxicity, Cognition recovery

Posted Date: March 2nd, 2021

DOI: https://doi.org/10.21203/rs.3.rs-247363/v1 
License: (c) (i) This work is licensed under a Creative Commons Attribution 4.0 International License. Read Full License

Version of Record: A version of this preprint was published at Chinese Medicine on July 28th, 2021. See the published version at https://doi.org/10.1186/s13020-021-00477-4. 


\section{Abstract}

Background: Synaptic damage and glutamate excitotoxicity have been implicated in the pathogenesis of vascular dementia (VD). Clathrin, RAB5B and N-methyl-d-aspartic acid receptor 1 (NMDAR1) proteins play a vital role in endocytosis of synaptic vesicles in neurons and glutamate over accumulation. Previous researches have been confirmed that Shenzhi Jiannao (SZJN) formula has an anti-apoptotic and neuroprotective effect in VD, but the underlying mechanisms are still unclear. In this study, we aimed to explore the effect of SZJN formula on cognitive impairment and glutamate excitotoxicity via clathrinmediated endocytosis (CME) in vivo and in vitro.

Methods: Both common carotid arteries were permanent occluded (2-vessel occlusion, 2VO) in male Sprague Dawley (SD) rats to model VD. One day after operation, the rats began daily treatment with SZJN formula for two weeks. The neuroprotective effects of SZJN formula was subsequently assessed by the novel object recognition test, Morris water maze, hematoxylin-eosin (HE) staining and Nissl staining. Glutamate cytotoxicity was assessed by detecting cell viability and cell death of PC12 cells. Immunohistochemistry, immunofluorescence, Western blot, and quantitative real-time PCR were used to detect the expression levels of clathrin, RAB5B, and NMDAR1.

Results: Administration of SZJN formula effectively improved short-term memory and spatial memory. SZJN treatment significantly reduced hippocampal neuronal loss, and recovered the arrangement and morphology of neurons and Nissl bodies. Moreover, SZJN formula promoted the proliferation of PC12 cells and inhibited glutamate-induced cell death. The down-regulation of clathrin and RAB5B, as well as the upregulation of NMDAR1 in the brain induced by 2VO or glutamate was also notably reversed by SZJN formula at both the protein and mRNA levels, which may contribute to SZJN formula induced improved neurological function.

Conclusions: Taken together, our findings provide evidence that the neuroprotective effects of SZJN formula in experimental VD maybe mediated through promoting the expression of clathirn-mediated endocytosis and reducing NMDARs-associated glutamate excitotoxicity. SZJN formula serves as a promising alternative therapy and may be a useful herbal medicine for preventing progression of VD.

\section{Introduction}

Vascular dementia (VD) is the second most common type of senile dementia and maybe the main dementia type in East Asia [1]. Globally, there are about 17 million cases of dementia suffering from VD at an annual cost of up to $\$ 200$ billion [2]. The pathogenesis of VD is closely related to glutamateinduced excitotoxicity and the impairment of glutamatergic vesicle trafficking $[3,4]$. Glutamate is the most important excitatory neurotransmitter in the central nervous system which could regulate synaptic transmission, synaptic plasticity and cognitive abilities [5,6]. Studies have confirmed that the excessive release of glutamate in the synaptic cleft and subsequent $\mathrm{Ca}^{2+}$ influx via NMDA-subtype glutamate 
receptors could lead to an intracellular cascade of cytotoxic events [7, 8]. The excessive activation of NMDA receptors evokes the impairment of synaptic plasticity, neuronal dysfunction and death $[9,10]$.

The endocytosis of synaptic vesicles, especially glutamatergic vesicle trafficking, is of great importance in the nervous system $[11,12]$. It has been demonstrated that the dysfunction of vesicle signaling at the end of axons could lead to neurosynaptic damage, abnormal dendritic structures, and eventually neuronal apoptosis [13]. CME is the major route for receptor internalization at the plasma membrane, in which clathrin as well as RAB5B are two key molecules responsible for the formation of synaptic vesicles, endocytosis, synaptic recycling, and release of neurotransmitter vesicles [14-16]. Additionally, the temporal cortex samples from VD patients showed significantly lower levels of synaptic-related proteins, especially the expression level of clathrin [17]. Although the exact mechanism of glutamate transmission remains unknown, evidence has been proved that clathrin-mediated endocytosis (CME) plays an important role in internalization of NMDA receptors $[18,19]$. The injury of CME process would delay the clearance of extracellular glutamate and cause glutamate toxicity in the brain. Hence, strategies to promote clathrin-mediated endocytosis of NMDA receptors to reduce glutamate neurotoxicity and recovery of synaptic plasticity may be of significant interest for the development of future therapies in VD.

SZJN formula, an important Chinese prescription, has been used for the treatment of chronic cerebral hypoperfusion and dementia. It is composed of Panax ginseng C.A.Mey., Anemarrhena asphodeloides Bunge, and Paeonia anomala subsp. veitchii (Lynch) D.Y.Hong \& K.Y.Pan, which have been officially recorded in the Chinese Pharmacopoeia 2020. The optimal ratio of these three herbs is 1:3:3 [20]. Pharmacological studies have shown that Panax ginseng C.A.Mey. has the function of regulating neurotransmitters levels, inhibiting hippocampal neuron apoptosis, and attenuating cerebral ischemia injury and cognitive deficits [21-24]. Anemarrhena asphodeloides Bunge has been verified to have cholinesterase inhibitory activity, and it could inhibit neuroinflammation and remove oxygen free radicals to improve learning and memory abilities $[25,26]$. Paeoniae Radix Rubra has also been demonstrated to have inhibitory effects on thrombus formation, as well as anti-inflammatory and antioxidative properties to reduce neurotoxic injury [27-29]. In our previous studies, we showed that SZJN formula has potent neuroprotective effects through regulation of neurotransmitters levels, inhibition of apoptotic cell death, and improving learning and memory deficits in VD models [30-32]. However, it is still not clear how SZJN formula inhibits glutamate neurotoxicity in VD models.

In this study, we observed the effect of SZJN formula on clathrin-mediated endocytosis and the toxic accumulation of glutamate in vivo and in vitro, and further explored its possible mechanism of learning and memory function improvement.

\section{Materials And Methods}

\section{Animals}


Adult male Sprague Dawley (SD) rats $(250 \pm 20 \mathrm{~g})$ were purchased from Beijing Vital River Laboratory Animal Technology Co., Ltd (certificate number: SCXK (Beijing) 2016-0006). The animals were housed with temperature $\left(22 \pm 1^{\circ} \mathrm{C}\right)$, humidity $(50 \pm 5 \%)$, and light (12-h light/dark cycle) control with free access to water and food. All experiments were conducted after being approved by the Institutional Animal Ethical Care and Use Committee of the Beijing University of Chinese Medicine (Approval ID: BUCM-42017121725-4025).

\section{Animal surgical procedure}

VD model was established by permanent bilateral common carotid artery occlusion (2VO) as described before [33]. Briefly, rats were anesthetized by intraperitoneal injection of $2 \%$ sodium pentobarbital (45 $\mathrm{mg} / \mathrm{kg}$ ). The neck of the rats was disinfected, and an incision was made to expose and separate the bilateral common carotid arteries along the cervical anterior median. Then, the bilateral blood vessels were ligated with 5-0 silk thread. In the sham-surgery group, the same surgery was performed with the exception of arterial ligation. The rats' body temperature was maintained at about $37 \pm 1^{\circ} \mathrm{C}$ during surgery.

\section{Experimental design}

SZJN formula is composed of $3 \mathrm{~g}$ Panax ginseng C.A.Mey., 9g Anemarrhena asphodeloides Bunge, and 9g Paeonia anomala subsp. veitchii (Lynch) D.Y.Hong \& K.Y.Pan. All herbs were pharmacopoeia-grade and prepared into granules by Beijing Tcmages Pharmaceutical Co., Ltd. (Beijing, China). Details of herbal materials are listed in Supplementary Fig. 1. The material basis of SZJN formula was determined using high-performance liquid chromatography (HPLC) and infrared spectrum (IS) analysis for monitoring the quality control purposes (Supplementary Fig. 2). Ginsenoside Rg1, ginsenoside Re, ginsenoside Rb1, Mangiferin, and paeoniflorin (the purities of all standards were higher than $98 \%$ by high-performance liquid chromatography analysis) were purchased from Beijing Solarbio Biotech Co., Ltd. (Beijing, China). The granules were suspended with distilled water to a final concentration of $38 \mathrm{~g} / \mathrm{L}$.

Rats were randomly divided into control group, sham-surgery group, VD group, SZJN group, and memantine hydrochloride (MH, H.Lundbeck A/S, Denmark) group. Rats in the SZJN group and MH group were intragastrically administered with SZJN formula (dissolved with distilled water, $0.2 \mathrm{~g} / \mathrm{kg}$ ), and memantine hydrochloride (dissolved with distilled water, $2.1 \mathrm{mg} / \mathrm{kg}$ ) one day after surgery for 14 days, respectively [31]. In the control group, sham-surgery group and VD group, rats were administered with equivalent distilled water intragastrically once per day for 14 days. Fig. 1A presents a schematic timeline of experiments.

\section{PC12 cell culture and experimental design}

Differentiated PC12 cells (Procell Life Science \& Technology Co., Ltd., Wuhan, China) were maintained in RPMI 1640 (Procell) supplemented with 10\% (v/v) fetal bovine serum (Procell)) and an antibiotic mixture of penicillin and streptomycin (1\%, Procell) in a humidified incubator at $37^{\circ} \mathrm{C}$ and $5 \% \mathrm{CO}_{2}$. When these 
cells reached $70 \%-80 \%$ confluence, they were trypsinized and subcultured. After seeding onto poly-Llysine-coated 96 - or 6-well plates at 3 cells/well for $24 \mathrm{~h}$, the cells were exposed to glutamate (final concentration: $22.5 \mathrm{mM}$, Sigma, USA) and incubated in the presence or absence of various concentrations of SZJN formula (final concentrations: $0.05,0.1$ and $0.2 \mathrm{mg} / \mathrm{mL}$ ) and memantine hydrochloride $(\mathrm{MH}, 10 \mu \mathrm{M})$ for $24 \mathrm{~h}$. The control cells were not administered any test agent or glutamate as the vehicle control. The glutamate-exposed cells were treated with glutamate for $24 \mathrm{~h}$ alone. The concentrations of SZJN formula in this experiment were selected according to our preliminary experiment (Supplementary Fig. 3). All experiments were replicated three times.

\section{Cell death and morphology changes}

To observe cell death and morphology changes during intervention process, the live-cell Essen Bioscience IncuCyte imaging system was used. Briefly, PC12 cells cultured by different concentrations of test agents were exposed to $5 \%$ YOYO $^{\mathrm{TM}}-1$ lodide solution, a cell impermeable dye that only enters cells with compromised membranes. The samples were observed under the IncuCyte imaging system at 10x magnification, which records both phase-contrast as well as fluorescent images over time.

\section{Novel object recognition (NOR) test}

NOR test was performed on the $8^{\text {th }}$ day of treatment as previously described [34]. During the training period, the exploration time for two identical objects was recorded within $5 \mathrm{~min}$. One day later, one object was changed to a different color and shape but with the same size. The exploration time for the new object and the familiar one was recorded within $5 \mathrm{~min}$. Rats were evaluated for their memory by expressing a preference for exploring the novel object [35]. Preference for the novel object was expressed as a discrimination index (DI, equation below).

$$
\mathrm{DI}=\frac{\text { time exploring the novel object-time exploring the familiar object }}{\text { time exploring the novel object+time exploring the familiar object }}
$$

\section{Morris water maze (MWM) test}

To assess spatial learning and memory, MWM test was performed on the $10^{\text {th }}$ day of treatment according to a procedure described with some modifications [36]. The circular water maze pool was $120 \mathrm{~cm}$ in diameter and divided into 4 quadrants. Each rat was allowed to search for the platform within $60 \mathrm{~s}$. When the rats failed to locate the submerged platform, they were manually directed toward the platform and allowed to remain on it for $10 \mathrm{~s}$. Each rat was trained 4 times a day for 4 consecutive days. On the $5^{\text {th }}$ day, a spatial probe trail was conducted by removing the platform from the pool. The rats were monitored to swim freely for $60 \mathrm{~s}$. The escape latency, total swimming distance, and percentage time spent in the target quadrant were recorded. All rats were kept from a motor impairment.

\section{Histological and pathological assessment}


Rats were anesthetized using $2 \%$ sodium pentobarbital administered intraperitoneally $(45 \mathrm{mg} / \mathrm{kg}$ ) and transcardially perfused with normal saline $(50-100 \mathrm{~mL})$ at $37^{\circ} \mathrm{C}$ and then perfused with $4 \%$ paraformaldehyde $(100 \mathrm{~mL})$ for $30 \mathrm{~min}$. The brain was immersion fixed in $4 \%$ paraformaldehyde for $48 \mathrm{~h}$ after which the brains were embedded in paraffin. A series of adjacent $3 \mu \mathrm{m}$ thick coronal sections were cut and employed for various staining. Additional hippocampal tissues were frozen in liquid nitrogen and stored at $-80^{\circ} \mathrm{C}$ for further analysis. For hematoxylin-eosin (H\&E) staining and Nissl staining, slices were dehydrated with $\mathrm{EtOH}(70 \%, 95 \%, 100 \%, 5$-min each), until xylene (5-min), followed by staining using hematoxylin-eosin as well as toluidine blue, respectively. Histopathological changes in the hippocampi were observed using an Olympus microscope (BX53, Japan).

\section{Immunohistochemistry}

Brain coronal sections were incubated for $1 \mathrm{~h}$ at room temperature with $10 \%$ goat serum then overnight at $4^{\circ} \mathrm{C}$ with primary antibodies to clathrin, RAB5B and NMDAR1 (Supplementary Table 1). After incubation with secondary antibodies for $50 \mathrm{~min}$, immunostaining was detected with 3,3'-diaminobenzidine kit (DAB, G1211, Servicebio). Image pro plus 6.0 image analysis software (Media Cybernetics, Rockville, MD, USA) was used for data analysis. The Area and Integral Optical Density (IOD) of the positive regions were imaged and the relative protein expression levels were calculated as the mean optical density (IOD/Area).

\section{Immunofluorescence}

PC12 cells were rinsed with PBS and fixed with $95 \%$ ethanol or $4 \%$ paraformaldehyde. After blocked with $3 \% \mathrm{BSA}$ for $1 \mathrm{~h}$ at $37^{\circ} \mathrm{C}$, cells were incubated with primary antibodies (Supplementary Table 1 ) for $2 \mathrm{~h}$ at $37^{\circ} \mathrm{C}$. Subsequently, cells were washed and incubated with secondary antibodies in the dark for $1 \mathrm{~h}$ at $37^{\circ} \mathrm{C}$, followed by staining with $20 \mu \mathrm{g} / \mathrm{mL}$ Hoechst 33342 (Sigma-Aldrich) for $15 \mathrm{~min}$. A IN Cell Analyzer high connotation cell imaging analysis system (2500HS, GE, USA) was used the observation.

\section{Western blot analysis}

Plasma membrane proteins were separated based on the Plasma Membrane Protein Isolation Kit (SM005 , Invent) protocol. The extracted protein of cells or tissues was determined for concentration with a BCA kit (Servicebio). Equal amounts of protein were electrophoresed on $10 \%$ polyacrylamide gels and then transferred onto a polyvinylidene fluoride (PVDF) membrane. After blocking with $5 \%$ skim milk for 1 $h$, the membranes were incubated overnight at $4^{\circ} \mathrm{C}$ with primary antibodies directed against clathrin, RAB5B, NMDAR1, and $\beta$-actin (Supplementary Table 1). The membranes were incubated with secondary antibodies at room temperature for $1 \mathrm{~h}$ and visualized using an ECL substrate (WBKLS0100, Millipore). FlourChem software (Alpha Innotech, FlourChem) was used to analyze the ratio of the gray value of each band to the $\beta$-actin band for relative expression levels. All samples were analyzed independently and in triplicate.

\section{Quantitative real-time PCR}


Total RNA was extracted from tissue samples and cells using a HiPure Total RNA Mini Kit (R4111-02, Magen) following the manufacturer instructions. After reverse transcription using the RevertAid First Strand cDNA Synthesis Kit (Thermo Fisher Scientific, USA), cDNA was used as the template for quantitative RT-PCR and performed using the real-time fluorescence quantitative PCR instrument (CFX96, Bio-Rad). The PCR cycle was as follows: $95^{\circ} \mathrm{C}$ pre-denaturation for $10 \mathrm{~min}, 95^{\circ} \mathrm{C}$ denaturation for $15 \mathrm{~s}$, $60^{\circ} \mathrm{C}$ annealing for $1 \mathrm{~min}$, with a total of 40 cycles. $\beta$-actin was designated as the internal reference gene and the relative expression level of target genes was calculated by the method. All samples and assays were performed in triplicate. Primers (Sangon Biotech, Shanghai, China) for clathrin, NMDAR1, RAB5B, and $\beta$-actin are listed in Supplementary Table 2.

\section{Statistical analysis}

SPSS 25.0 software was used for data analysis. Data were expressed as mean \pm SD. MWM data were analyzed using two-way repeated measure ANOVA. For the others were analyzed by one-way ANOVA followed by post hoc test (Tukey's tests). If the variance of statistics was uneven, a Nonparametric test was performed. $P$ values $<0.05$ was considered statistically significant.

\section{Results}

\section{SZJN formula ameliorates cognitive deficits in VD rats}

One week after the administration of SZJN formula, animals were tested in a series of behavioral experiments including NOR test and MWM test cognition and memory ability. In 2 days of the NOR test, the discrimination index was decreased in the VD group compared with the sham-surgery group $(P<$ 0.01). In contrast, VD rats treated with SZJN formula or MH exhibited significantly higher discrimination index indicating improved short-term memory, in comparison to VD model rats $(P<0.01)$. no significant difference was found between the control and sham-surgery groups $(P>0.05)$ (Fig. 1B). In the MWM test, the escape latency of the VD group was significantly longer than that of the sham-surgery group on day $1,2,3$, and $4(P<0.01$, Fig. 1C), during the probe navigation training. On the contrary, the escape latency of SZJN and MH groups was significantly shorter compared with the VD group $(P<0.01, P<0.05$, Fig. 2C). The total distance in SZJN and MH groups decreased significantly, compared with VD group $(\mathrm{P}<$ 0.01 , Fig. 1D). After the hidden platform was removed, the time spent in the target quadrant of the SZJN group was significantly higher than that of the VD group $(P<0.05$, Fig. 1E). Likewise, $\mathrm{MH}$ treatment yielded the similar improvements but there was no significant difference compared to the model group ( $P$ $>0.05$, Fig. 2E). In addition, the swimming speed was not significantly different between each group ( $P>$ 0.05 , Fig. 1F), indicating no motor function impairment in all the groups. These data indicate that both the SZJN formula and MH improves cognitive function and memory in VD rats.

\section{SZJN formula administration alleviates neuronal damage and death in the hippocampus of VD rats}

H\&E and Nissl staining was performed to determine the neuroprotective effects of SZJN formula on the hippocampal CA1 region of brain tissues. As shown in Fig. 2A, neurons were arranged tightly and orderly 
with clear nuclei in the hippocampus of the control and sham-surgery group. However, obvious pathological abnormalities with scattered neurons arrangement, neurons shrunken and loss, or dark color staining were observed significantly in the VD group ( $P<0.01$, Fig. 2A and C). In comparison, both SZJN formula and $\mathrm{MH}$ alleviated these histopathological alterations (Fig. 2A). Additionally, SZJN formula or $\mathrm{MH}$ treatment for 2 weeks caused remarkable reduction in neurons loss in the hippocampal CA1 of rats, compared to the VD group $(P<0.01$, Fig. 2 C).

For Nissl staining, neurons exhibit a normal morphology with distinct round or oval nuclei and nucleoli, and clear Nissl bodies in the cytoplasm in the control and sham-surgery groups (Fig. 2B). In comparison, a great number of apoptotic neurons, with Nissl body fragmentation, cell loss and karyorrhexis were observed in the VD group. The number of Nissl-positive cells significantly decreased in the hippocampal CA1 region of VD rats, compared to the sham-surgery group $(P<0.01$, Fig. 2D). Compared with the VD group, administration of SZJN formula and $\mathrm{MH}$ clearly improved the morphology and arrangement of neurons, as well as markedly reversed 2 VO-induced neuronal loss in the CA1 region $(P<0.01, P<0.05$,

Fig. 2B and D).

\section{SZJN increases clathrin and RAB5B expression, whereas reduces cytomembrane NMDAR1 expression in the hippocampus of VD rats}

We evaluated whether SZJN formula could inhibit glutamate toxicity and promote the CME process associated with upregulating clathrin and RAB5B, and downregulating NMDAR1 in the hippocampus. Immunohistochemical staining of the hippocampus illustrated that the expressions of clathrin and RAB5B were decreased significantly in VD rats $(P<0.01)$, while increased in the SZJN group $(P<0.01$, Fig. 3A-C). The activation of NMDAR1 was significantly increased in VD rats $(P<0.01)$, while decreased in the SZJN group $(P<0.01$, Fig. $3 A$ and $\mathbf{D})$. Western blot analysis showed similar results as the immunohistochemistry. The expressions of clathrin, RAB5B and cytoplasmic NMDAR1 in the hippocampus were increased dramatically in the VD group compared with the sham-surgery group $(P<$ 0.01, Fig. 3E-H). However, administration with SZJN formula showed a remarkable increasing of clathrin , RAB5B and cytoplasmic NMDAR1 expression compared with the VD group $(P<0.01)$. The expression of cytomembrane NMDAR1 was significantly increased in VD rats $(P<0.01)$, while decreased in the SZJN group $(P<0.01$, Fig. 3E and I).

qRT-PCR showed that the mRNA level of clathrin was reduced in the VD group compared with the shamsurgery group $(P<0.01)$. In contrast, rats in the SZJN group showed obviously increased mRNA level of clathrin in comparison to those in the VD group $(P<0.01$, Fig. $3 \mathbf{J})$. Moreover, the mRNA expression of NMDAR1 increased in the hippocampus of VD rats compared to the sham-surgery rats $(P<0.01)$. However, SZJN formula decreased the expression of NMDAR1 notably in the hippocampus $(P<0.01$, Fig. $3 \mathrm{~K})$.

\section{SZJN formula rescued the VD cell model from glutamate-induced PC12 cell damage}


The results (Fig. 4A) revealed that compared with the control group, the viability of the PC12 cells treated with SZJN formula alone with the concentrations of $0.05,0.1$, and $0.2 \mathrm{mg} / \mathrm{mL}$ was significantly increased, whereas SZJN formula with 0.025 and $0.4 \mathrm{mg} / \mathrm{mL}$ did not show the significance of promoting cell proliferation. Among the effective concentrations, 0.05 and $0.1 \mathrm{mg} / \mathrm{mL}$ of SZJN formula showed the best enhancing cell viability effect. Thus, SZJN formula at the concentrations of $0.05,0.1$, and 0.2 $\mathrm{mg} / \mathrm{mL}$ was selected for use in the subsequent experiments. Then the concentration-dependent response of glutamate-induced cytotoxicity was determined. As shown in Fig. 4B, PC12 cell viability was slightly enhanced by glutamate with $22 \mathrm{mM}$, and inhibited by glutamate with 22.5 and $23 \mathrm{mM}$ markedly. Besides, cells cultured with $22 \mathrm{mM}$ glutamate did not show obvious green fluorescent area, whereas $22.5 \mathrm{mM}$ glutamate showed 3 times and $23 \mathrm{mM}$ glutamate showed 6 times of that in the control group by $24 \mathrm{hpt}$ (Fig. 4C). Thus, glutamate at the concentration of $22.5 \mathrm{mM}$ was selected for use in the subsequent experiments, combined with the screening results in Supplementary Fig. 3.

As shown in Fig. 4D, stimulation with glutamate alone resulted in a significant decrease in cell viability as compared with the control group by $24 \mathrm{hpt}$. The number of dead cells and green fluorescent intensity in the glutamate group were about 3 times of that in the control group by $24 \mathrm{hpt}$ (Fig. 4E and F). Similarly, the green fluorescent area in the glutamate group increased by $0 \mathrm{hpt}$ and increased to over $250000 / \mu \mathrm{m}^{2}$ per image by $24 \mathrm{hpt} \mathrm{(Fig.} \mathrm{4G).} \mathrm{On} \mathrm{the} \mathrm{contrary,} \mathrm{treatment} \mathrm{with} \mathrm{SZJN} \mathrm{formula} \mathrm{in} \mathrm{the} \mathrm{presence} \mathrm{of} \mathrm{glutamate}$ exhibited a significant decrease of glutamate-induced toxicity in a concentration under $0.05,0.1$, and 0.2 $\mathrm{mg} / \mathrm{mL}$ of SZJN formula. SZJN formula could reduce the number of dead cells, green fluorescent intensity and area, as well as recovered cell viability (Fig. 4D-G). Among the effective concentrations, 0.1 and $0.2 \mathrm{mg} / \mathrm{mL}$ of SZJN formula showed the best neuroprotective effect, as shown in Fig. 4E-G. Moreover, representative fluorescent images of cell morphological changes at 0 and 24 hpt are shown in Fig. 4H.

\section{SZJN formula enhances clathrin and RAB5B expressions and reduces NMDAR1 expression in glutamate- induced PC12 cells}

To further explore whether the clathrin mediated glutamate synaptic vesicle transport was also regulated by SZJN formula in vitro, the protein and mRNA levels of clathrin, RAB5B, and NMDAR1 in glutamate induced PC12 cells were investigated. Immunofluorescence assay showed that the expressions of clathrin and RAB5B proteins were much lower and NMDAR1 expression was higher after treatment with glutamate $(P<0.01)$. After treatment with SZJN formula for 24 hours, clathrin and RAB5B protein expressions were enhanced, as well as NMDAR1 expression was declined compared with the glutamate group $(P<0.05$, Fig. 5A-F).

Western blot analysis showed the similar results with Immunofluorescence. The expression of clathrin and RAB5B were markedly decreased $(P<0.01)$, but significantly elevated with SZJN formula treatment in a dose-dependent manner $(P<0.01$, Fig. 6A-C). Meanwhile, after glutamate exposure, the expression of NMDAR1 was drastically elevated in the glutamate group as compared with the control group $(P<0.01)$, but down-regulated by SZJN formula in a dose-dependent manner $(P<0.01$, Fig. 6A and $\mathbf{D})$. In addition, 
clathrin and NMDAR1 levels were also reversed by MH treatment $(P<0.05)$, but RAB5B level had no significant difference ( $P>0.05$, Fig. 6A-D). Moreover, qRT-PCR demonstrated that the mRNA level of clathrin decreased and NMDAR1 mRNA level increased in glutamate group $(P<0.01)$. SZJN formula not only enhanced clathrin gene expression $(P<0.01$, Fig. 6E) but also attenuated NMDAR1 expression in glutamate-induced PC12 cells $(P<0.05$, Fig. 6F). These findings were consistent with the Western blot results. Collectively, SZJN formula might repress glutamate toxicity partially by modulating the expression of NMDAR1 and CME molecules.

\section{Discussion}

In the present study, we demonstrate that SZJN formula could reduce the neurons damage, promote cell proliferation and attenuate cognitive deficits both in VD rats and cell models. Additionally, SZJN formula down-regulated NMDAR1 expression level, as well as activated the key molecules of the endocytosis process such as clathrin and RAB5B, suggesting that the CME of NMDA receptors may involve in the inhibitory effects of SZJN formula on glutamate excitotoxicity, which might account for a prosurvival effect and cognitive improvement of SZJN formula.

Cerebral ischemia hypoperfusion injury impaired the ability of working memory and spatial learning and memory. Emerging evidence has suggested that SZJN formula administration significantly recovered the impaired spatial memory induced by hypoxia and ameliorated the hippocampus-dependent memory dysfunction induced by cyclophosphamide and scopolamine [32,37]. However, it is still unknown whether SZJN formula could attenuate cognitive deficits in VD rats. In this study, SZJN formula and memantine hydrochloride administration reduced the escape latency as well as the total distance and prolonged the time in the target quadrant, suggesting that SZJN formula treatment inhibited the spatial memory impairment induced by $2 \mathrm{VO}$. These results were consistent with previous studies and indicated that SZJN formula showed the cognition-enhancing effects on VD.

Numerous studies have demonstrated that cerebral ischemia-reperfusion results in hippocampal injury, which is a contributor to cognition decline [38, 39]. The Nissl body is a normal structure in the cytoplasm of neurons and has the function of protein synthesis in neurons [40]. Nissl body fragmentation could be investigated when injured by cerebral ischemia. Our previous researches suggested that SZJN formula is effective in inhibiting apoptosis, enhancing the concentrations of neurotransmitters, repairing mitochondrial function, and improving cerebral blood flow [31, 37]. In this study, our results showed $2 \mathrm{VO}$ injury caused obvious pathological abnormalities manifested as loosed arranged neurons, destroyed Nissl bodies, cell degeneration, or dark color staining in the hippocampal CA1 region. However, SZJN formula significantly reversed neuron damage. Moreover, SZJN formula inhibited the glutamate-induced PC12 cell damage and death. The findings in present result are consistent with previous studies, suggesting SZJN formula has a neuroprotective effect against vascular dementia.

The pathogenesis of VD includes various aspects, such as neuronal apoptosis, oxidative stress, and neurotransmitter abnormities. In particular, many studies have mentioned that glutamate neurotoxicity 
cannot be ignored in the development of cognitive impairment with VD [41,42]. Studies have shown that neuronal death induced by cerebral ischemia and reperfusion is mainly associated with the high concentrations of glutamate and excessive activation of NMDAR $[43,44]$. Glutamate overaccumulation leads to glutamatergic neurotransmitter dysfunction, signal transmission disorder, and oxidative stress injury. During the early stages of ischemia and hypoxia, NMDA receptors expression in the hippocampal CA1 region of VD rats increases, which aggravates the excitotoxic effect of glutamate. Excessive glutamate continues to stimulate postsynaptic NMDA receptors, resulting in over activation of NMDA receptors and excessive $\mathrm{Ca}^{2+}$ influx, ultimately resulting in calcium overload, neurons injury, and cognitive dysfunction. A previous study has demonstrated that reducing glutamate release and inhibiting NMDA receptors activity could inhibit $\mathrm{Ca}^{2+}$ influx and alleviate the cytotoxic injury [45]. Besides, as the most important subunit structure of NMDA receptors, NMDAR1 is responsible for synaptic transmission and plasticity. A growing number of studies suggest that the loss of NMDAR1 or the mutations in genes that encode NMDAR1 results in the total loss of NMDAR function, is associated with many neuropsychiatric disorders [46-48]. mutant NMDAR1 subunit has reduced glycine sensitivity, which is correlated with reduced forward trafficking of NMDARs to the cell surface [49]. In the present study, we found that SZJN formula could prominently reduce the protein and mRNA expression levels of NMDAR1. These results suggested that SZJN formula may decline the overaccumulation of glutamate to alleviate glutamate neurotoxicity.

The over activation of NMDA receptors could not only lead to the $\mathrm{Ca}^{2+}$ influx, but also affect the internalization mediated by clathrin. A study investigated that the NMDA-evoked $\mathrm{Ca}^{2+}$ influx could affect the expression of clathrin, the synaptic protein that plays an important role in synaptic vesicular formation and transport of glutamate and other excitatory amino acids [50]. Clathrin, mediating the classical endocytosis, is widely involved in signal transduction of various physiological activities, including nutrient absorption, cell growth and differentiation, synaptic transmission, and immune response. Binding to NMDAR1 receptors of postsynaptic membrane needs the mediation of clathrin and the internalization of NMDA receptors also relies on clathrin [51,52]. Several studies have reported that the process of endocytosis is critical for NMDAR mediated excitotoxicity. Excessive activation of NMDAR triggered in increased endocytosis, which may be an important step in NMDAR mediated excitotoxic neuronal death $[53,54]$. The apoptosis induced by over activation of NMDAR In Drosophila melanogaster was inhibited by dynamin, which could block the endocytosis mediated by clathrin [55]. Besides, clathrin is pivotal for the fusion of synaptic vesicles and presynaptic membranes. Once synaptic vesicles lose their endocytosis function, the area around the presynaptic membranes increase. This will result in a gradual decrease in lateral membrane tension, which consequently cause neurons to lose their normal synaptic functions $[56,57]$. Studies have shown that several disease-related genes can damage and interfere with vesicle endocytosis, which can then result in decreased levels of endocytosis-related proteins such as clathrin, AP-2, and dynamin [58-60]. RAB5B is a small ATPase molecule that has been shown as a regulator in the early endocytic pathway of cell surface NMDARs [10]. It has been investigated that the internalization of NMDAR1 was accompanied by increased RAB5B expression 16 . Moreover, other studies have shown that the clathrin-mediated endocytosis is also involved in amyloid- $\beta$ 
and a-synuclein clearance or transmission. For instance, a-synuclein oligomerization promoted NMDAR1 subunit internalization [61]. Amyloid- $\beta$ could decrease the surface expression of NMDA receptors by promoting endocytosis of receptor proteins [62]. Our study showed that SZJN formula could significantly up-regulate clathrin and RAB5B expression, and we speculated that was associated with the significant reduction of surface NMDAR1 expression, which may be closely involved in promoting endocytosis of NMDARs and attenuating toxic glutamate accumulation.

The present study uncovered that SZJN formula could attenuate neurons damage and cognitive deficits in VD, and the role of SZJN formula in the reducing glutamate cytotoxicity may be related to the regulation of the key molecules of clathrin mediated endocytosis of NMDAR receptors. However, there are several limitations needing to be improved in our study. Firstly, we did not measure the level of glutamate in vivo, as well as the expression of clathrin combined with NMDA receptors on the membrane. Secondly, we only found that SZJN formula could regulate these classical endocytosis molecules, but whether SZJN formula ameliorated the cognitive function through this pathway were not verified. In the future, more studies can be carried out to explore the specific mechanism of SZJN formula on clathrin mediated NMDA receptors endocytosis in VD.

\section{Conclusions}

In summary, we demonstrated that SZJN formula significantly attenuated neuronal damage, glutamate cytotoxicity and cognitive deficits in VD, which was probably related to the regulation of the glutamate synaptic vesicle transport. Enhancement of clathrin and RAB5B expression may be involved in the internalization of NMDA receptors mediated by CME after SZJN formula treatment. These results suggest that SZJN formula may be a potential therapeutic agent for the prevention and treatment of VD. Additional experimental and clinical studies will be required to assess the potential for clinical application of SZJN formula.

\section{Abbreviations}

SZJN formula: Shenzhi Jiannao formula; VD: Vascular dementia; NMDAR1: N-methyl-D-aspartic acid receptor 1; CME: Clathrin-mediated endocytosis; HPLC: High-performance liquid chromatography; IS: Infrared spectrum; PVDF: Polyvinylidene fluoride.

\section{Declarations}

\section{Acknowledgments}

We would like to extend our gratitude to Professor Zhen-Yun Han from the Shenzhen Hospital of Beijing University of Chinese Medicine, Shenzhen, China, for providing valuable suggestions and revising the manuscript.

\section{Authors' contributions}


Study design: ZYH, DFT; experimental implementation: DFT, YYG, DDZ, QG, ZC, GLL, YCW; data analysis: DFT, YYG, JFL, RS; material contribution and equipment coordination: ZYH; paper writing: DFT. All authors have read and approved the final version of the manuscript.

\section{Funding}

This work was supported by the National Natural Science Foundation of China, No. 81673910 (to ZYH). The funding sources had no role in study conception and design, data analysis or interpretation, paper writing or deciding to submit this paper for publication.

\section{Availability of data and materials}

The data used to support the current study are available from the corresponding author on reasonable request.

\section{Ethics approval and consent to participate}

This study was approved by the Institutional Animal Ethical Care and Use Committee of the Beijing University of Chinese Medicine (Approval ID: BUCM-4-2017121725-4025), and the experimental procedures were conducted in accordance with the ethical standards of the Chinese Association of Accreditation of Laboratory Animal Care.

\section{Consent for publication}

Not applicable.

\section{Competing interests}

The authors declare that they have no competing interests.

\section{Author details}

${ }^{1}$ Beijing University of Chinese Medicine, Beijing, 100029, China. ${ }^{2}$ Department of Scientific Research, Beijing Hospital of Traditional Chinese Medicine, Capital Medical University, Beijing 100010, China.

${ }^{3}$ Shenzhen Hospital of Beijing University of Chinese Medicine (Longgang), Shenzhen, 518172, China.

\section{References}

1. Iadecola C. The pathobiology of vascular dementia. Neuron. 2013;80(4):844-66.

2. Smith EE. Clinical presentations and epidemiology of vascular dementia. Clinical science (London, England : 1979). 2017;131(11):1059-68.

3. Busse M, Kunschmann R, Dobrowolny H, Hoffmann J, Bogerts B, Steiner J, et al. Dysfunction of the blood-cerebrospinal fluid-barrier and N-methyl-D-aspartate glutamate receptor antibodies in dementias. European archives of psychiatry and clinical neuroscience. 2018;268(5):483-92. 
4. Zhang S, Yan ML, Yang L, An XB, Zhao HM, Xia SN, et al. MicroRNA-153 impairs hippocampal synaptic vesicle trafficking via downregulation of synapsin I in rats following chronic cerebral hypoperfusion. Experimental neurology. 2020;332:113389.

5. Revett TJ, Baker GB, Jhamandas J, Kar S. Glutamate system, amyloid ß peptides and tau protein: functional interrelationships and relevance to Alzheimer disease pathology. Journal of psychiatry \& neuroscience : JPN. 2013;38(1):6-23.

6. Lin R, Li L, Zhang Y, Huang S, Chen S, Shi J, et al. Electroacupuncture ameliorate learning and memory by improving $\mathrm{N}$-acetylaspartate and glutamate metabolism in APP/PS1 mice. Biological research. 2018;51(1):21.

7. Sasaki-Hamada S, Suzuki A, Sanai E, Matsumoto K, Oka JI. Neuroprotection by chotosan, a Kampo formula, against glutamate excitotoxicity involves the inhibition of GluN2B-, but not GluN2Acontaining NMDA receptor-mediated responses in primary cultured cortical neurons. Journal of pharmacological sciences. 2017;135(3):134-7.

8. Liu Z, Qiu X, Mak S, Guo B, Hu S, Wang J, et al. Multifunctional memantine nitrate significantly protects against glutamate-induced excitotoxicity via inhibiting calcium influx and attenuating $\mathrm{PI3K} /$ Akt/GSK3beta pathway. Chemico-biological interactions. 2020;325:109020.

9. Madeira C, Vargas-Lopes C, Brandão CO, Reis T, Laks J, Panizzutti R, et al. Elevated Glutamate and Glutamine Levels in the Cerebrospinal Fluid of Patients With Probable Alzheimer's Disease and Depression. Frontiers in psychiatry. 2018;9:561.

10. Yu W, Yang W, Li X, Li X, Yu S. Alpha-synuclein oligomerization increases its effect on promoting NMDA receptor internalization. International journal of clinical and experimental pathology. 2019;12(1):87-100.

11. Du X, Li J, Li M, Yang X, Qi Z, Xu B, et al. Research progress on the role of type I vesicular glutamate transporter (VGLUT1) in nervous system diseases. Cell \& bioscience. 2020;10:26.

12. Silm K, Yang J, Marcott PF, Asensio CS, Eriksen J, Guthrie DA, et al. Synaptic Vesicle Recycling Pathway Determines Neurotransmitter Content and Release Properties. Neuron. 2019;102(4):786800.e5.

13. Chang RY, Nouwens AS, Dodd PR, Etheridge N. The synaptic proteome in Alzheimer's disease. Alzheimer's \& dementia : the journal of the Alzheimer's Association. 2013;9(5):499-511.

14. Kirchhausen T, Owen D, Harrison SC. Molecular structure, function, and dynamics of clathrinmediated membrane traffic. Cold Spring Harbor perspectives in biology. 2014;6(5):a016725.

15. Robinson MS. Forty Years of Clathrin-coated Vesicles. Traffic (Copenhagen, Denmark). 2015;16(12):1210-38.

16. Wood LA, Larocque G, Clarke NI, Sarkar S, Royle SJ. New tools for "hot-wiring" clathrin-mediated endocytosis with temporal and spatial precision. The Journal of cell biology. 2017;216(12):4351-65.

17. Gallart-Palau X, Serra A, Qian J, Chen CP, Kalaria RN, Sze SK. Temporal lobe proteins implicated in synaptic failure exhibit differential expression and deamidation in vascular dementia. Neurochemistry international. 2015;80:87-98. 
18. Nong Y, Huang YQ, Ju W, Kalia LV, Ahmadian G, Wang YT, et al. Glycine binding primes NMDA receptor internalization. Nature. 2003;422(6929):302-7.

19. Pai B, Siripornmongcolchai T, Berentsen B, Pakzad A, Vieuille C, Pallesen S, et al. NMDA receptordependent regulation of miRNA expression and association with Argonaute during LTP in vivo. Frontiers in cellular neuroscience. 2014;7:285.

20. Yang AR, Tian X, Zhong H, Fan JP. The best combination of effective screening component of SZJN tablet (In Chinese). Chinese Archives of Traditional Chinese Medicine. 2009;27(12):2600-2.

21. Zhu JD, Wang JJ, Zhang XH, Yu Y, Kang ZS. Panax ginseng extract attenuates neuronal injury and cognitive deficits in rats with vascular dementia induced by chronic cerebral hypoperfusion. Neural regeneration research. 2018;13(4):664-72.

22. Lin J, Gao S, Wang T, Shen Y, Yang W, Li Y, et al. Ginsenoside Rb1 improves learning and memory ability through its anti-inflammatory effect in $A \beta(1-40)$ induced Alzheimer's disease of rats. American journal of translational research. 2019;11(5):2955-68.

23. Liu M, Bai X, Yu S, Zhao W, Qiao J, Liu Y, et al. Ginsenoside Re Inhibits ROS/ASK-1 Dependent Mitochondrial Apoptosis Pathway and Activation of Nrf2-Antioxidant Response in Beta-AmyloidChallenged SH-SY5Y Cells. Molecules (Basel, Switzerland). 2019;24(15).

24. Wang B, Zhu Q, Man X, Guo L, Hao L. Ginsenoside Rd inhibits apoptosis following spinal cord ischemia/reperfusion injury. Neural regeneration research. 2014;9(18):1678-87.

25. Sun Y, Peng Y, Li LG, Zheng LW, Lin DJ, Li LZ, et al. A Platform for Screening Potential Anticholinesterase Fractions and Components Obtained from Anemarrhena asphodeloides Bge for Treating Alzheimer's Disease. Evidence-based complementary and alternative medicine : eCAM. 2014;2014:524650.

26. Wang Z, Cai J, Fu Q, Cheng L, Wu L, Zhang W, et al. Anti-Inflammatory Activities of Compounds Isolated from the Rhizome of Anemarrhena asphodeloides. Molecules (Basel, Switzerland). 2018;23(10).

27. Xie P, Cui L, Shan Y, Kang WY. Antithrombotic Effect and Mechanism of Radix Paeoniae Rubra. BioMed research international. 2017;2017:9475074.

28. Li B, Yang ZB, Lei SS, Su J, Pang MX, Yin C, et al. Beneficial Effects of Paeoniflorin Enriched Extract on Blood Pressure Variability and Target Organ Damage in Spontaneously Hypertensive Rats. Evidence-based complementary and alternative medicine : eCAM. 2017;2017:5816960.

29. Xiong S, Wang Y. Simultaneous determination of paeoniflorin from total glucosides of paeony in Sprague-Dawley rats and spontaneously hypertensive rats by high-performance liquid chromatography-tandem mass spectrometry: in vivo and in vitro studies. Biomedical chromatography : BMC. 2016;30(11):1766-71.

30. X. MK, Y. HZ, Y. MD, R. S, Fan JP, Zhang FS. Effect of Shenzhi Jiannao Capsule on Glu and GABA in cortex and hippocampus of vascular dementia rat model. Liaoning Journal of traditional Chinese Medicine. 2016;43(11):2421-4. 
31. Ma KX, Han ZY, Zhang HYJ, Ma DY, Su R, Zhang FS. Effect of SZJN Capsule on regional cerebral blood flow in rats with cerebral ischemia (In Chinese). Jilin Traditional Chinese Medicine. 2018;38(01):72-5.

32. Ma DY, Han ZY, Fan JP. ShenzhiJiannao Capsule on central cholinergic system of scopolamine induced memory impairment mice (In Chinese). Chinese Journal of traditional Chinese medicine information. 2012;19(05):47-8+55.

33. Zong W, Zeng X, Chen S, Chen L, Zhou L, Wang X, et al. Ginsenoside compound K attenuates cognitive deficits in vascular dementia rats by reducing the $A \beta$ deposition. Journal of pharmacological sciences. 2019;139(3):223-30.

34. Karasawa J, Hashimoto K, Chaki S. D-Serine and a glycine transporter inhibitor improve MK-801induced cognitive deficits in a novel object recognition test in rats. Behavioural brain research. 2008;186(1):78-83.

35. Lueptow LM. Novel Object Recognition Test for the Investigation of Learning and Memory in Mice. Journal of visualized experiments : JoVE. 2017(126).

36. Bhuvanendran S, Bakar SNS, Kumari Y, Othman I, Shaikh MF, Hassan Z. Embelin Improves the Spatial Memory and Hippocampal Long-Term Potentiation in a Rat Model of Chronic Cerebral Hypoperfusion. Scientific reports. 2019;9(1):14507.

37. Wu Q, Zhang SJ, Zhang YC, Xing EL, Yang AR, Hu JH, et al. Study on the protective mechanism of ShenzhiJiannao Recipe on brain mitochondrial damage in rats with hypoxia induced cognitive impairment (In Chinese). World traditional Chinese medicine. 2017;12(10):2403-7.

38. Zhu Y, Li S, Liu J, Wen Q, Yu J, Yu L, et al. Role of JNK Signaling Pathway in Dexmedetomidine PostConditioning-Induced Reduction of the Inflammatory Response and Autophagy Effect of Focal Cerebral Ischemia Reperfusion Injury in Rats. Inflammation. 2019;42(6):2181-91.

39. Guan X, Li X, Yang X, Yan J, Shi P, Ba L, et al. The neuroprotective effects of carvacrol on ischemia/reperfusion-induced hippocampal neuronal impairment by ferroptosis mitigation. Life sciences. 2019;235:116795.

40. Wang SP, Wang D, Li HX, Liu L, Duan XH. Influence of miR-34a on cerebral neuronal apoptosis in rats with cerebral ischemia reperfusion through the Notch1 signaling pathway. European review for medical and pharmacological sciences. 2019;23(18):8049-57.

41. Ma S, Liu H, Jiao H, Wang L, Chen L, Liang J, et al. Neuroprotective effect of ginkgolide K on glutamate-induced cytotoxicity in PC 12 cells via inhibition of ROS generation and $\mathrm{Ca}(2+)$ influx. Neurotoxicology. 2012;33(1):59-69.

42. Oo EM, Ruamyod K, Khowawisetsut L, Turbpaiboon C, Chaisuksunt V, Uawithya P, et al. Germinated Brown Rice Attenuates Cell Death in Vascular Cognitive Impaired Mice and Glutamate-Induced Toxicity In HT22 Cells. Journal of agricultural and food chemistry. 2020;68(18):5093-106.

43. Wu Y, Chen C, Yang Q, Jiao M, Qiu S. Endocytosis of GluN2B-containing NMDA receptors mediates NMDA-induced excitotoxicity. Molecular pain. 2017;13:1744806917701921. 
44. Zhang N, Xing M, Wang $\mathrm{Y}$, Tao $\mathrm{H}$, Cheng $\mathrm{Y}$. Repetitive transcranial magnetic stimulation enhances spatial learning and synaptic plasticity via the VEGF and BDNF-NMDAR pathways in a rat model of vascular dementia. Neuroscience. 2015;311:284-91.

45. Zhang Y, Mao X, Lin R, Li Z, Lin J. Electroacupuncture ameliorates cognitive impairment through inhibition of $\mathrm{Ca}(2+)$-mediated neurotoxicity in a rat model of cerebral ischaemia-reperfusion injury. Acupuncture in medicine : journal of the British Medical Acupuncture Society. 2018;36(6):401-7.

46. Traynelis SF, Wollmuth LP, McBain CJ, Menniti FS, Vance KM, Ogden KK, et al. Glutamate receptor ion channels: structure, regulation, and function. Pharmacological reviews. 2010;62(3):405-96.

47. Lemke JR, Geider K, Helbig KL, Heyne HO, Schütz H, Hentschel J, et al. Delineating the GRIN1 phenotypic spectrum: A distinct genetic NMDA receptor encephalopathy. Neurology. 2016;86(23):2171-8.

48. Chen W, Shieh C, Swanger SA, Tankovic A, Au M, McGuire M, et al. GRIN1 mutation associated with intellectual disability alters NMDA receptor trafficking and function. Journal of human genetics. 2017;62(6):589-97.

49. Skrenkova K, Hemelikova K, Kolcheva M, Kortus S, Kaniakova M, Krausova B, et al. Structural features in the glycine-binding sites of the GluN1 and GluN3A subunits regulate the surface delivery of NMDA receptors. Scientific reports. 2019;9(1):12303.

50. Wang J, Lv X, Wu Y, Xu T, Jiao M, Yang R, et al. Postsynaptic RIM1 modulates synaptic function by facilitating membrane delivery of recycling NMDARs in hippocampal neurons. Nature communications. 2018;9(1):2267.

51. Yang W, Yu W, Li X, Li X, Yu S. Alpha-synuclein differentially reduces surface expression of N-methyld-aspartate receptors in the aging human brain. Neurobiology of aging. 2020;90:24-32.

52. Hanley JG. The Regulation of AMPA Receptor Endocytosis by Dynamic Protein-Protein Interactions. Frontiers in cellular neuroscience. 2018;12:362.

53. Borsello T, Croquelois K, Hornung JP, Clarke PG. N-methyl-d-aspartate-triggered neuronal death in organotypic hippocampal cultures is endocytic, autophagic and mediated by the c-Jun $\mathrm{N}$-terminal kinase pathway. The European journal of neuroscience. 2003;18(3):473-85.

54. Malinow R, Malenka RC. AMPA receptor trafficking and synaptic plasticity. Annual review of neuroscience. 2002;25:103-26.

55. Kiselev A, Socolich M, Vinós J, Hardy RW, Zuker CS, Ranganathan R. A molecular pathway for lightdependent photoreceptor apoptosis in Drosophila. Neuron. 2000;28(1):139-52.

56. Kononenko NL, Haucke V. Molecular mechanisms of presynaptic membrane retrieval and synaptic vesicle reformation. Neuron. 2015;85(3):484-96.

57. Soykan T, Kaempf N, Sakaba T, Vollweiter D, Goerdeler F, Puchkov D, et al. Synaptic Vesicle Endocytosis Occurs on Multiple Timescales and Is Mediated by Formin-Dependent Actin Assembly. Neuron. 2017;93(4):854-66.e4.

58. Garcia MD, Formoso K, Aparicio GI, Frasch ACC, Scorticati C. The Membrane Glycoprotein M6a Endocytic/Recycling Pathway Involves Clathrin-Mediated Endocytosis and Affects Neuronal 
Synapses. Frontiers in molecular neuroscience. 2017;10:296.

59. Pan PY, Li X, Wang J, Powell J, Wang Q, Zhang Y, et al. Parkinson's Disease-Associated LRRK2 Hyperactive Kinase Mutant Disrupts Synaptic Vesicle Trafficking in Ventral Midbrain Neurons. The Journal of neuroscience : the official journal of the Society for Neuroscience. 2017;37(47):11366-76.

60. Fan F, Funk L, Lou X. Dynamin 1- and 3-Mediated Endocytosis Is Essential for the Development of a Large Central Synapse In Vivo. The Journal of neuroscience : the official journal of the Society for Neuroscience. 2016;36(22):6097-115.

61. Oh SH, Kim HN, Park HJ, Shin JY, Bae EJ, Sunwoo MK, et al. Mesenchymal Stem Cells Inhibit Transmission of a-Synuclein by Modulating Clathrin-Mediated Endocytosis in a Parkinsonian Model. Cell reports. 2016;14(4):835-49.

62. Juul Rasmussen I, Tybjærg-Hansen A, Rasmussen KL, Nordestgaard BG, Frikke-Schmidt R. Bloodbrain barrier transcytosis genes, risk of dementia and stroke: a prospective cohort study of 74,754 individuals. European journal of epidemiology. 2019;34(6):579-90.

\section{Figures}


A Sham-surgery/2VO

NOR test

MWM test

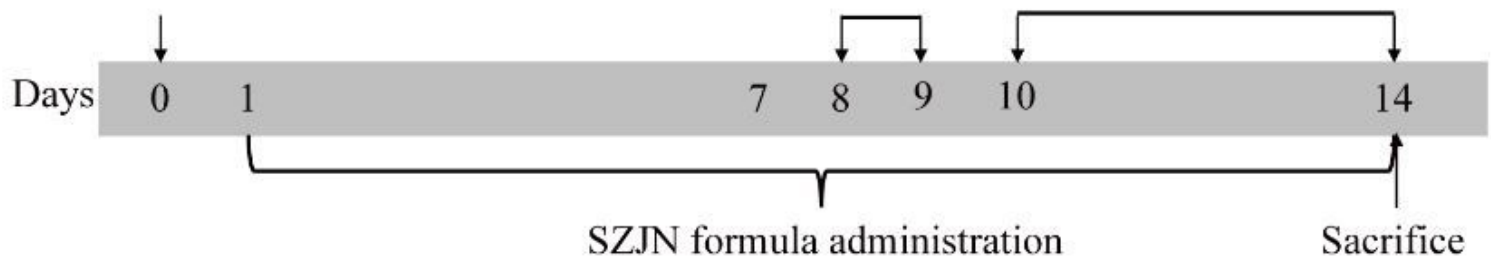

B

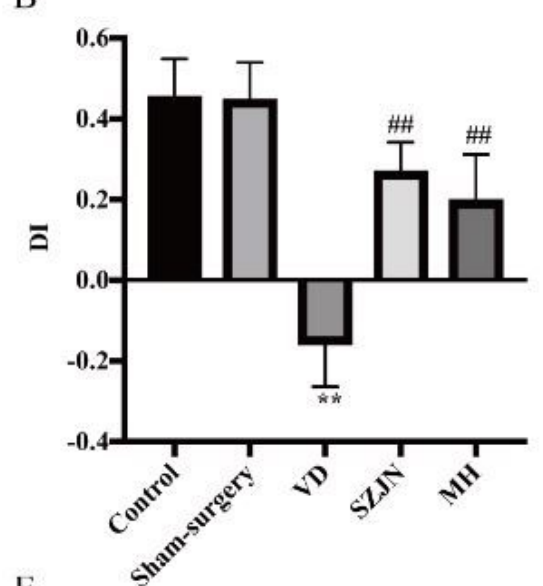

$\mathrm{E}$

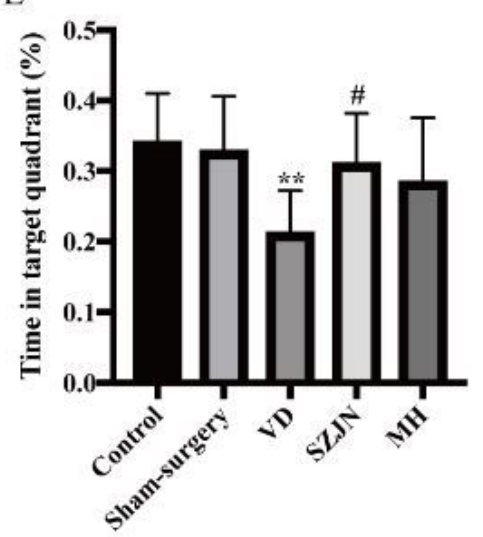

$\mathrm{C}$

C

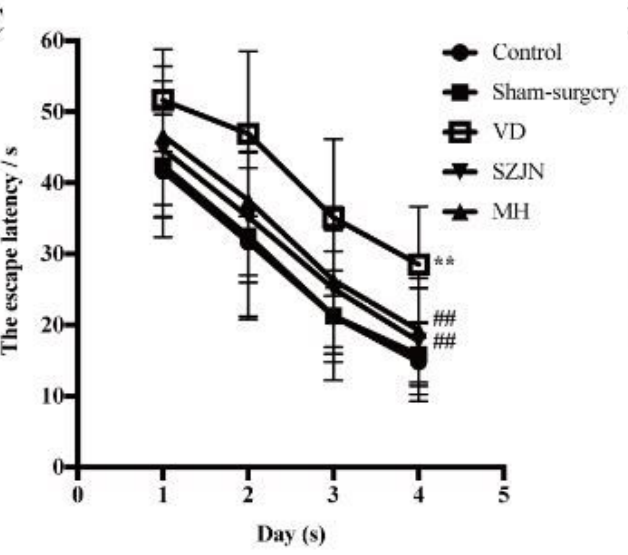

$\mathrm{F}$

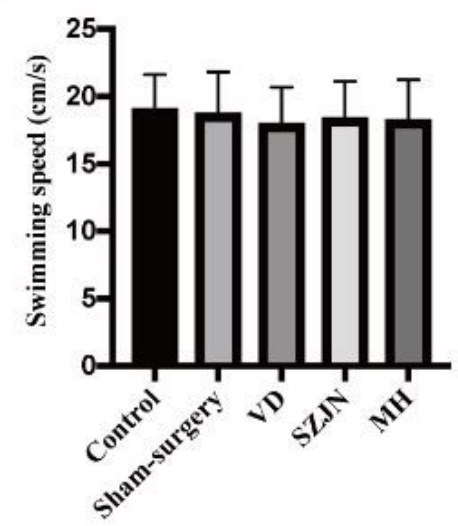

D

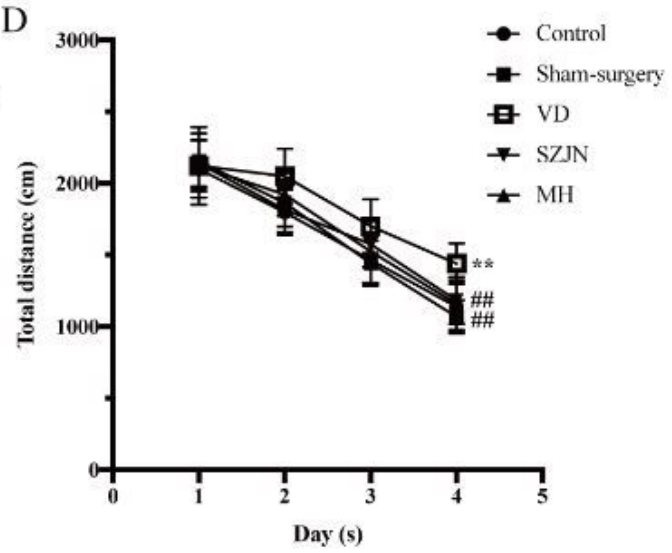

Figure 1

SZJN formula ameliorates cognitive deficits and improves memory abilities in VD rats. (A) Schematic timeline of the animal study. (B) Discrimination index (DI) of NOR test. (C) The escape latency and (D) total distance for reaching the hidden platform during 4 days in the probe navigation trial. (E) The time spent in the target quadrant and $(F)$ swimming speed in the spatial probe trial. Values are expressed as means $\pm S D(n=12)$. ${ }^{*}<0.05, * * P<0.01$ versus sham-surgery group. $\# P<0.05, \# \# P<0.01$ versus VD group. 


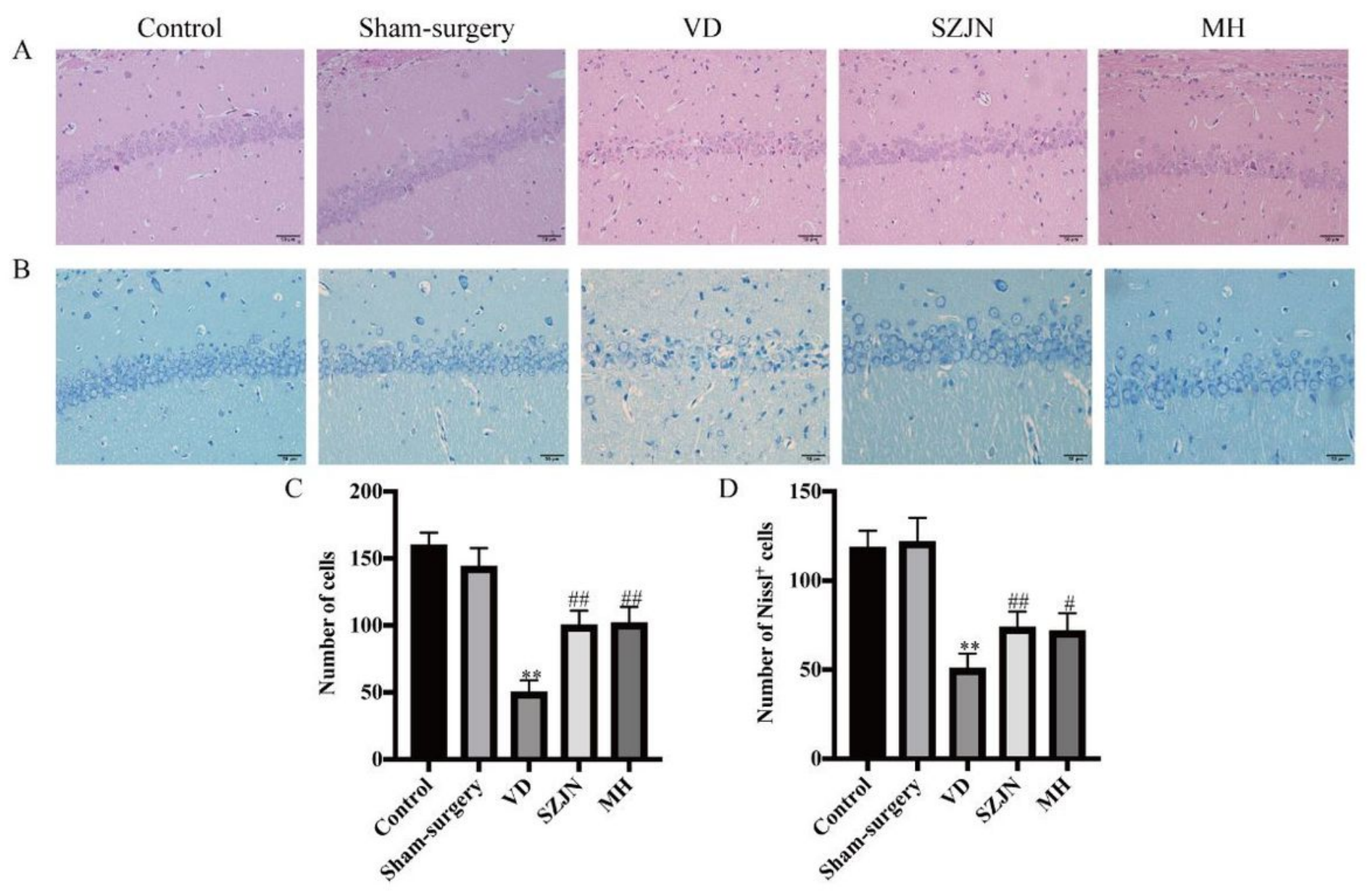

Figure 2

SZJN formula inhibited hippocampal neuronal damage and loss in VD rats. Representative images and quantitative graph of $(A, C) H \& E$ staining and $(B, D)$ Nissl staining in hippocampal CA1 region were performed as described in Methods $(400 \times)$. Values are expressed as means $\pm S D(n=6)$. ${ }^{*}<0.05$, **P< 0.01 versus sham-surgery group. $\# \mathrm{P}<0.05, \# \# \mathrm{P}<0.01$ versus VD group. 


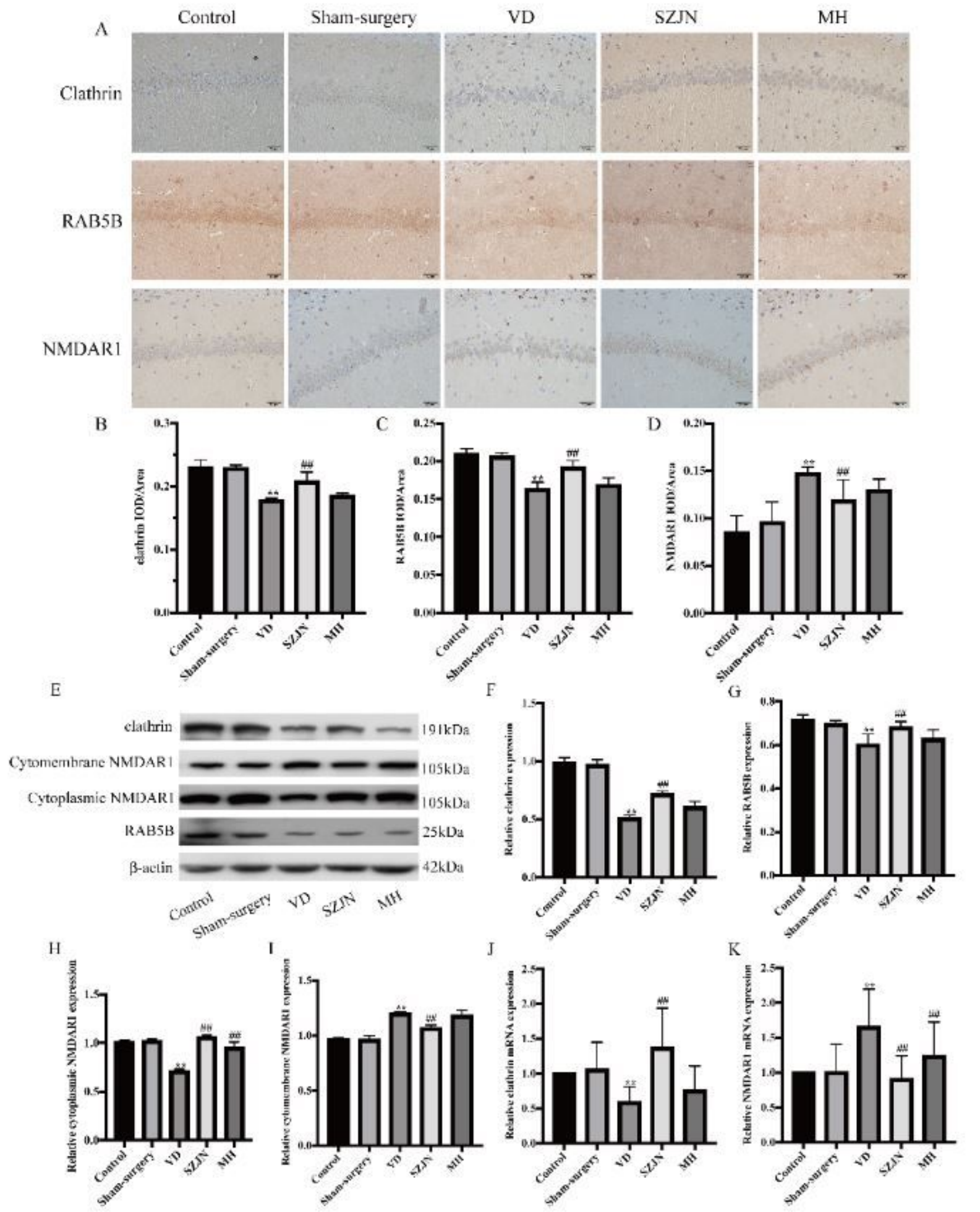

Figure 3

SZJN increases clathrin and RAB5B expression, whereas reduces cytomembrane NMDAR1 expression in the Hippocampus of VD Rats. (A) Immunohistochemical analysis of clathrin, RAB5B, and NMDAR1 expression in hippocampal CA1 regions $(400 \times)$. Quantitative analysis showed the expression of (B) clathrin, (C) RAB5B, and (D) NMDAR1 in hippocampal CA1 regions. (E) Western blot was performed to detect the levels of clathrin, RAB5B, cytoplasmic NMDAR1, and cytomembrane NMDAR1 in the 
hippocampus of VD rats. Quantitative analysis of $(F)$ clathrin, $(G)$ RAB5B, $(H)$ cytoplasmic NMDAR1, and (I) cytomembrane NMDAR1 expression, the densities of the bands were normalized with respect to the values of $\beta$-actin. qRT-PCR analyzed $(J)$ clathrin mRNA and (K) NMDAR1 mRNA expression levels. Values are expressed as means $\pm S D(n=6)$. ${ }^{*}<0.05, * \star P<0.01$ versus sham-surgery group. $\# P<0.05, \# \# P<$ 0.01 versus VD group.

A

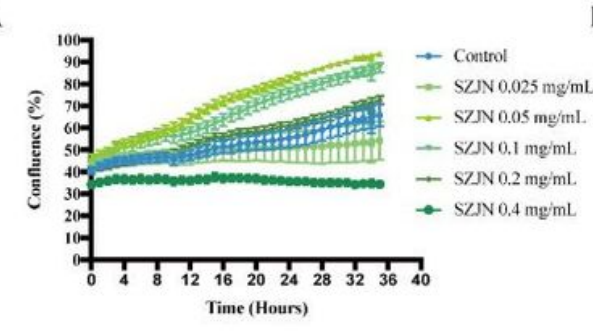

D

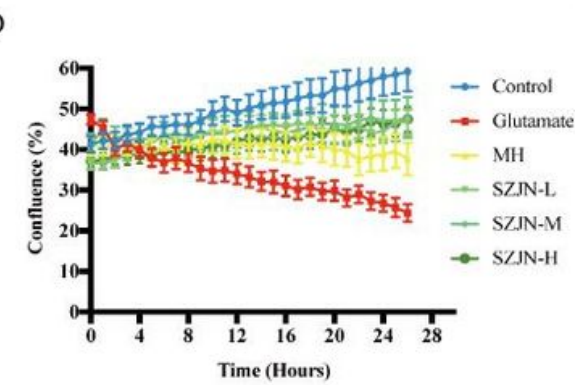$$
\text { G }
$$

G

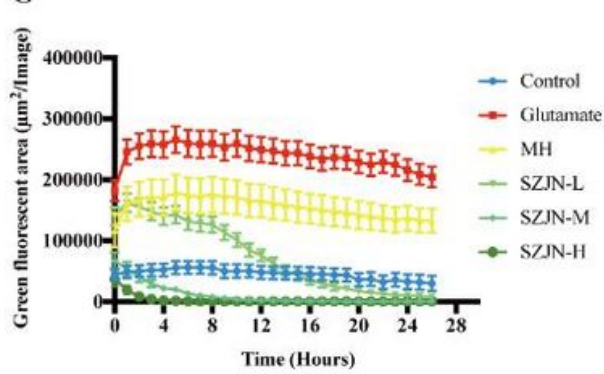

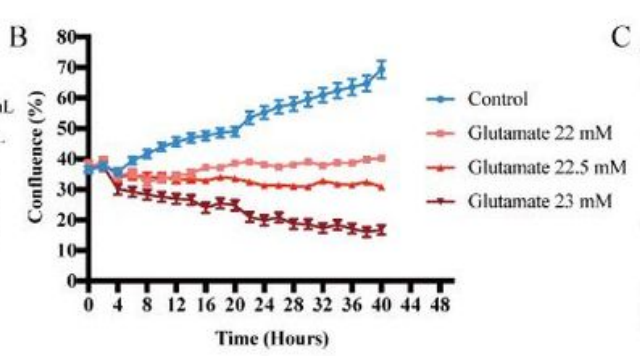

E

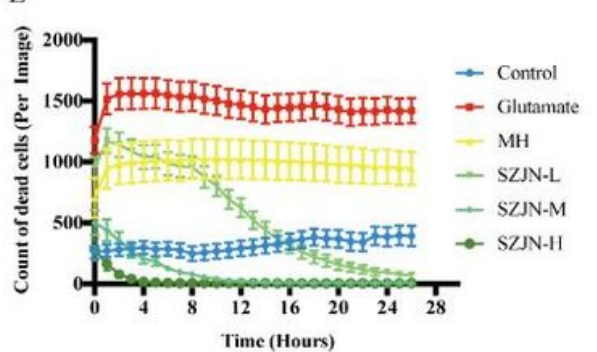

$\mathrm{H}$

"
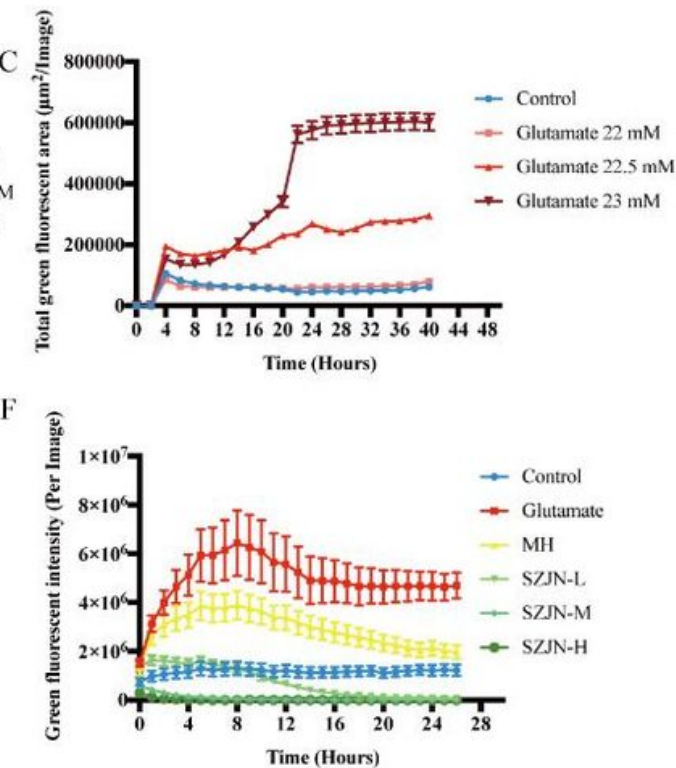

Control Glutamate $\mathrm{MH}$

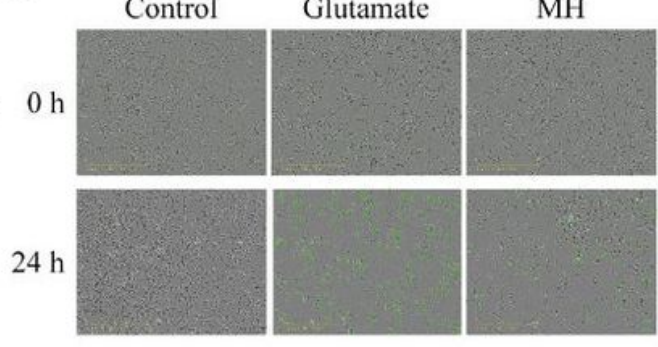

SZJN-L

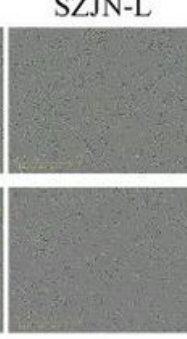

SZJN-M

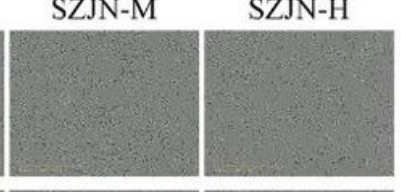

\section{Figure 4}

Effects of SZJN formula on glutamate-induced cytotoxicity to PC12 cells. (A) Effect of SZJN formula itself $(0.025,0.05,0.1,0.2$, and $0.4 \mathrm{mg} / \mathrm{mL})$ on basal growth of PC12 cells. (B) Effect of glutamate itself $(22,22.5$ and $23 \mathrm{mM})$ on viability of PC12 cells. (C) Effect of glutamate itself $(22,22.5$ and $23 \mathrm{mM})$ on the area of total green fluorescence. (D) Effect of SZJN formula on cell viability, (E) number of dead cells, (F) green fluorescent intensity, and (G) green fluorescent area in glutamate-exposed PC12 cells. Following treatment of the cells with various concentrations of SZJN formula $(0.05,0.1$, and $0.2 \mathrm{mg} / \mathrm{mL})$ for $24 \mathrm{~h}$ and exposure to $22.5 \mathrm{mM}$ of glutamate for $24 \mathrm{~h}$. $(\mathrm{H})$ Effects of SZJN formula in glutamate-induced cell morphological changes. SZJN-L: $0.05 \mathrm{mg} / \mathrm{mL}$, SZJN-M: $0.1 \mathrm{mg} / \mathrm{mL}, \mathrm{SZJN}-\mathrm{H}: 0.2 \mathrm{mg} / \mathrm{mL}$. Values are expressed as means $\pm S D(n=4)$. ${ }^{*} P<0.05$, ${ }^{\star *} P<0.01$ as compared with the control group. \#P<0.05, $\# \# \mathrm{P}<0.01$ as compared with the glutamate group. 

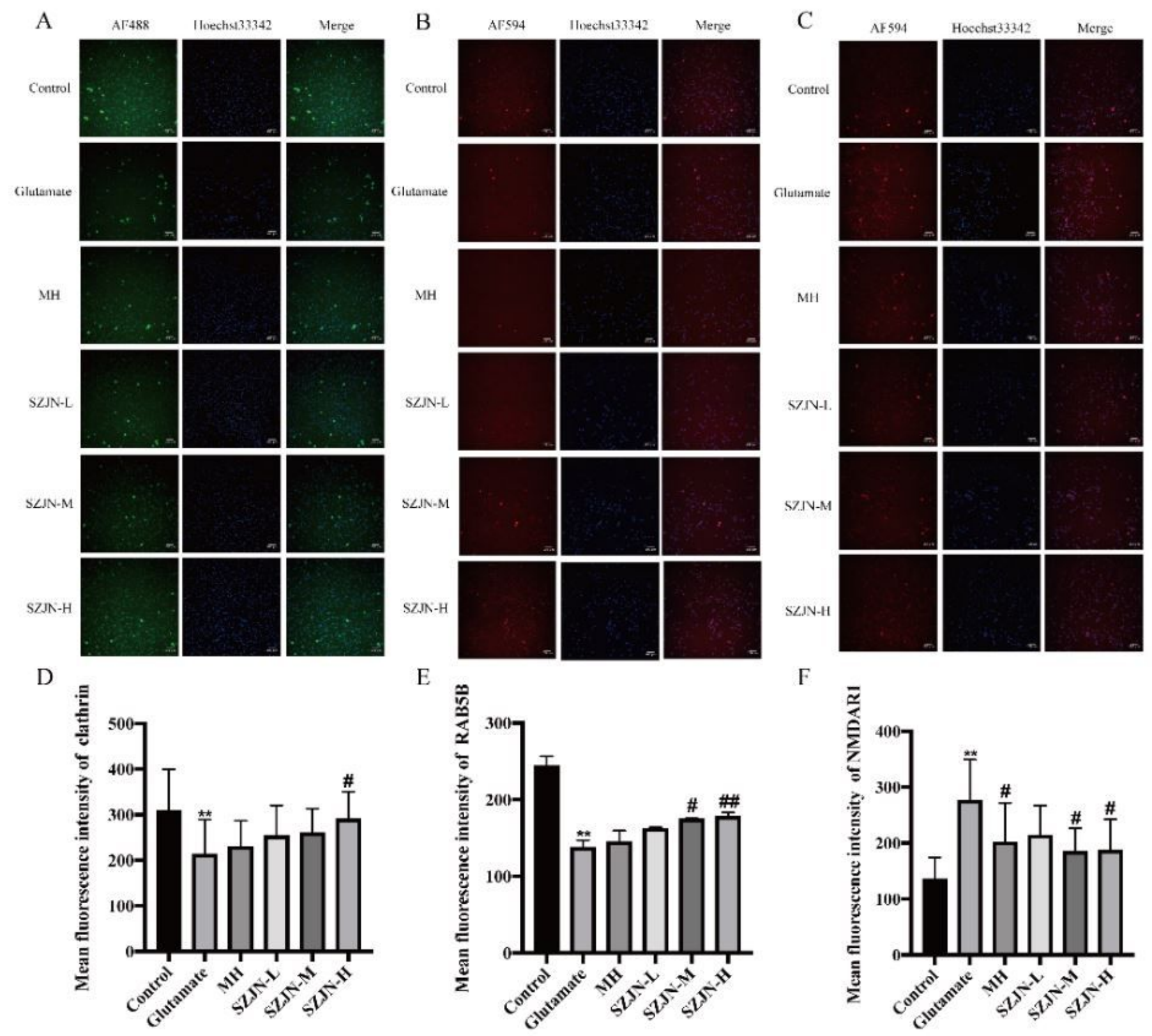

\section{Figure 5}

Effects of SZJN formula on the expression of clathrin, RAB5B, and NMDAR1 by using immunofluorescence. Representative photomicrographs of (A) clathrin (green, AF488), (B) RAB5B (red, AF594), and (C) NMDAR1 (red, AF594) immunofluorescence are shown. Nuclei were stained with Hoechst 33342 (blue). The graph show the mean fluorescence intensity of (D) clathrin, (E) RAB5B, and (F) NMDAR1 in PC12 cells. SZJN-L: $0.05 \mathrm{mg} / \mathrm{mL}$, SZJN-M: $0.1 \mathrm{mg} / \mathrm{mL}$, SZJN-H: $0.2 \mathrm{mg} / \mathrm{mL}$. Values are expressed as means $\pm S D(n=3)$. ${ }^{*}<<0.05$, ${ }^{\star * P}<0.01$ as compared with the control group. $\# P<0.05$, $\#$ \#P $<0.01$ as compared with the glutamate group. 
A

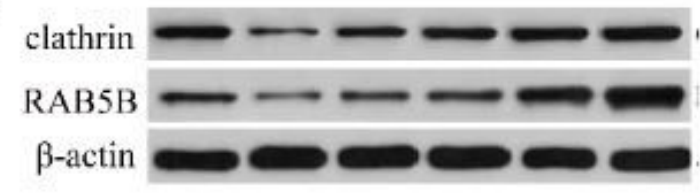

NMDAR 1

$\beta$-actin

Glutamate

$\begin{array}{rcccccc}\text { SZJNF } & - & - & - & 0.05 & 0.1 & 0.2 \\ \mathrm{MH} & - & - & + & - & - & -\end{array}$

$\mathrm{C}$

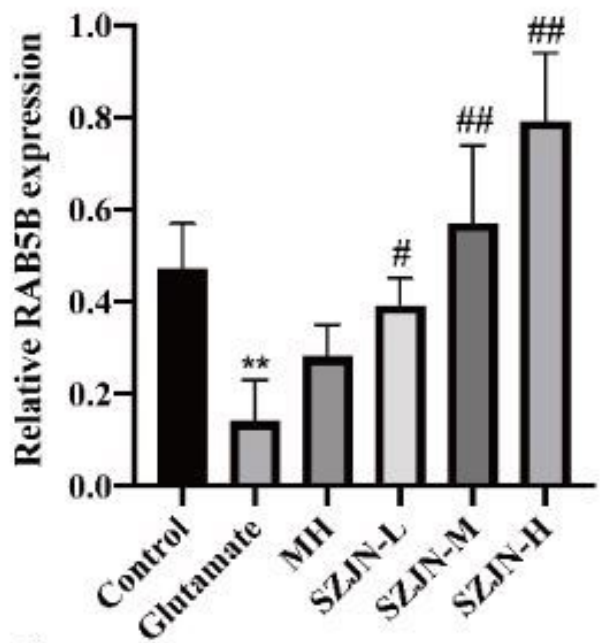

E

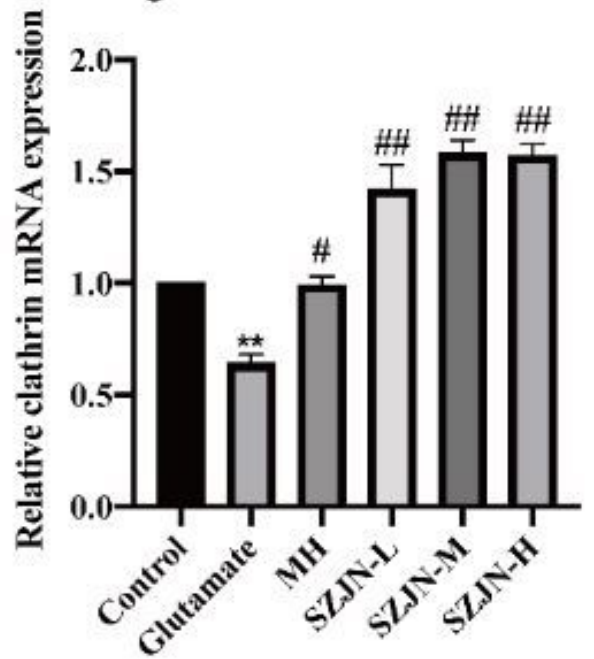

B

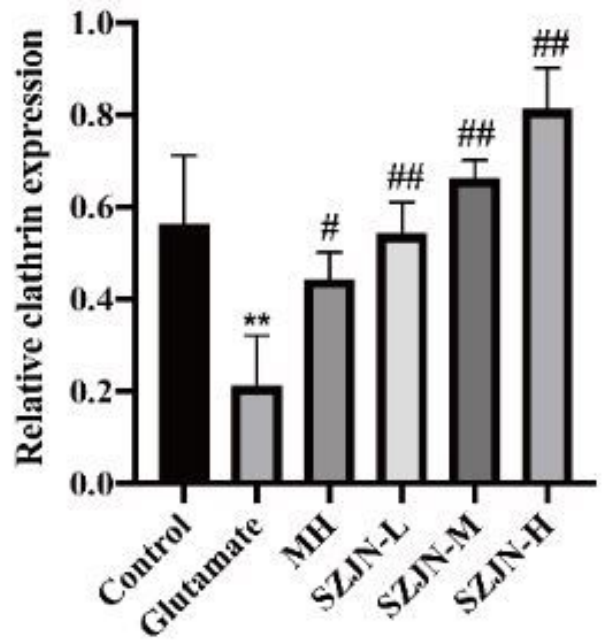

D

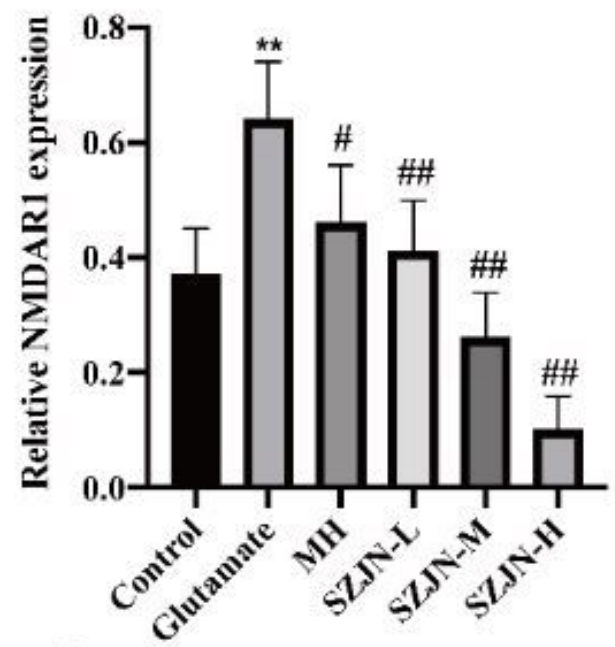

F

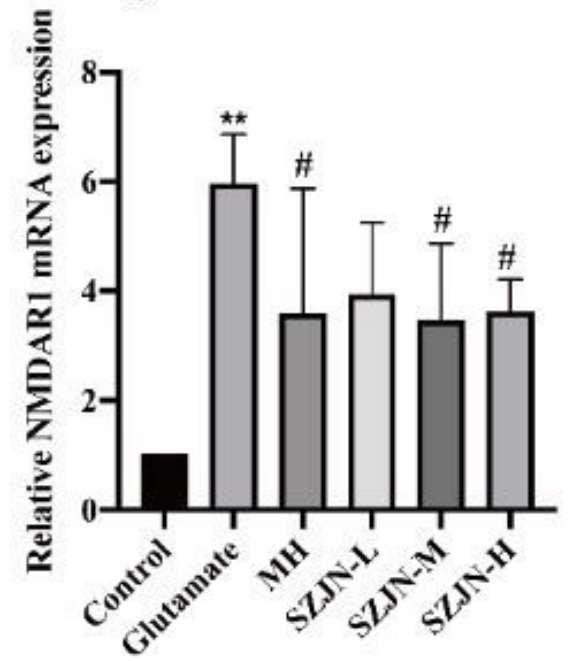

Figure 6

Effects of SZJN formula on the protein and mRNA expressions of clathrin, RAB5B, and NMDAR1. (A) Western blot was performed to detect the protein levels of clathrin, RAB5B, and NMDAR1 in glutamate treated PC12 cells. Quantitative analysis of (B) clathrin, (C) RAB5B, and (D) NMDAR1 expressions. $\beta$-Actin was used as the internal control. qRT-PCR analyzed (E) clathrin mRNA and (F) NMDAR1 mRNA expression levels. SZJN-L: $0.05 \mathrm{mg} / \mathrm{mL}$, SZJN-M: $0.1 \mathrm{mg} / \mathrm{mL}$, SZJN-H: $0.2 \mathrm{mg} / \mathrm{mL}$. Values are expressed 
as means $\pm S D(n=3) . * P<0.05, * * P<0.01$ as compared with the control group. $\# P<0.05, \# \# P<0.01$ as compared with the glutamate group.

\section{Supplementary Files}

This is a list of supplementary files associated with this preprint. Click to download.

- Supplementarymaterials.docx

- Fig.S1.tif

- Figs2.tif

- figures3.tif 